\title{
Comparison of Fluorescent Tag DNA Labeling Methods Used for Expression Analysis by DNA Microarrays
}

BioTechniques 33:620-630 (September 2002)

\author{
A. Richter, C. Schwager, S. \\ Hentze, W. Ansorge, M.W. \\ Hentze, and M. Muckenthaler \\ European Molecular Biology \\ Laboratory, Heidelberg, \\ Germany
}

\section{INTRODUCTION}

Expression profiling using cDNAor oligonucleotide-based microarrays is rapidly becoming a popular technique in the scientific community. Microarrays are a versatile tool to analyze gene expression levels on a genome-wide basis (8). Customized microarrays focus on a selection of genes that are involved in specific biochemical pathways or diseases (e.g., cancer) (4). cDNAs or oligonucleotides representing the genes of interest are immobilized on a solid support such as glass or nylon. To analyze the transcriptome of cells or tissues for the expression of these genes, the mRNA is extracted and cDNA probes are synthesized directly or indirectly by incorporating fluorescent or radioactive label into the cDNA. Probe synthesis protocols using fluorescent dyes rely on the labeling of an experimental and control cDNA sample by incorporating $\mathrm{Cy} 3$ or $\mathrm{Cy} 5$ dyes, respectively. Following synthesis, the two labeled cDNA pools are mixed and co-hybridized to the microarray, conferring the advantage that each microarray experiment is internally controlled for hybridization conditions. Following washing, the array is scanned with a confocal laser scanner and subsequently analyzed, determining the relative fluorescent intensities between the control and experimental samples for each gene that is represented on the microarray.

The establishment of DNA microarray technology ("chips") may vary from laboratory to laboratory. Some laboratories spot their own chips, while others buy ready-spotted microarrays and perform probe making, hybridiza- tions, and data evaluation themselves. It is also possible to send RNA samples for commercial evaluation on DNA microarrays and to receive a result file. In spite of the relative ease with which microarrays can be used today, it is important to consider the different protocols for any given step in microarray analysis. In particular, an understanding of how different methods for the preparation of fluorescent cDNA probes influence a gene expression pattern will be important when data from different laboratories need to be compared, as in public DNA microarray databases.

A successful microarray experiment must be both sensitive and accurate in the detection of a gene expression pattern. In particular, sensitivity is a top priority for many medical applications since the starting material (e.g., cellular aspirates or tissue biopsies) is usually obtained in limited amounts - often just enough for a single experiment. Here we report the comparative evaluation of five different commercial and/or published protocols for the synthesis of fluorescent cDNA probes, with respect to accuracy and sensitivity in detecting an established gene expression pattern. The protocols tested are based on different principles for fluorescent probe synthesis, which are represented in Figure 1.

In the direct labeling method (Figure 1A), a Cy3 or Cy5 fluorescently labeled nucleotide is directly incorporated during a first-strand cDNA synthesis starting from $20 \mu \mathrm{g}$ total RNA. The labeled cDNA is then hybridized to the attached DNA on the array. In the T7 RNA polymerase amplification method (Figure $1 B)$, the amount of input material needed is usually $5-10 \mu \mathrm{g}$ total RNA. A first- 
strand cDNA reaction is performed using a composite primer between oligo(dT) and the promoter sequence for T7 RNA polymerase. Following secondstrand synthesis, the dsDNA is used as a template for amplification by in vitro transcription using T7 RNA polymerase. The resulting antisense RNA is then used as template for the synthesis of fluorescently labeled cDNA primed with random hexamers. The aminoallyl labeling method (Figure 1C) works on the premise that the direct incorporation of $\mathrm{Cy} 3$ and Cy5 fluorescently labeled nucleotides during cDNA synthesis is not equally effective (1). To address this concern, aminoallyl modified nucleotides are incorporated during the first-strand cDNA reaction. In a subsequent step, $\mathrm{Cy} 3$ or $\mathrm{Cy} 5$ dyes are coupled to the reactive amino groups in the modified cDNA. The amount of total RNA required is $10-15 \mu \mathrm{g}$. In the hapten-antibody enzymatic labeling method (Figure 1D), fluorescein (used as a hapten for subsequent Cy3 labeling) or biotin (used as a hapten for subsequent Cy5 labeling) modified nucleotides are incorporated during first-strand cDNA synthesis. The modified cDNA is hybridized to the attached DNA of the microarray. After hybridization and stringent washing, two

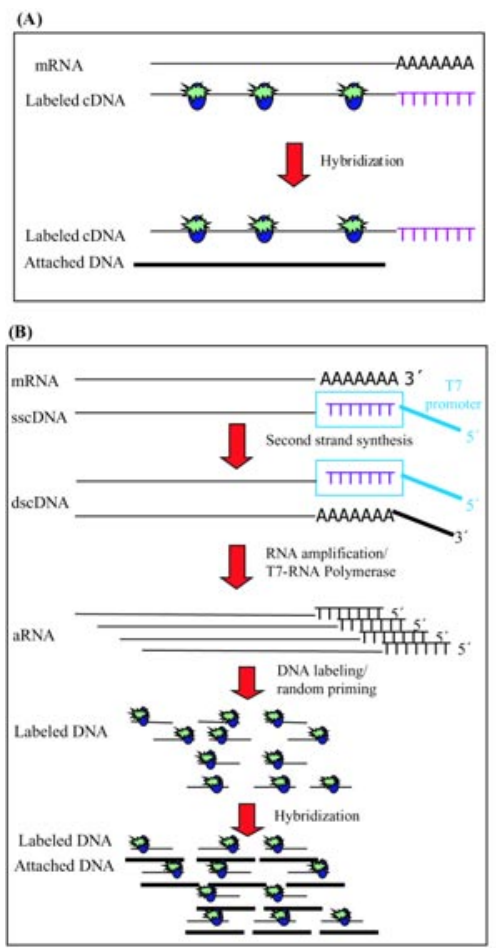

(C)

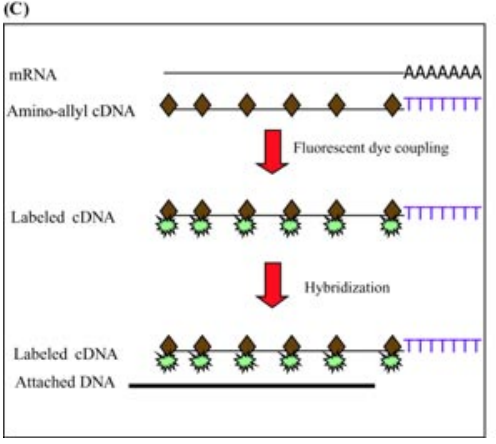

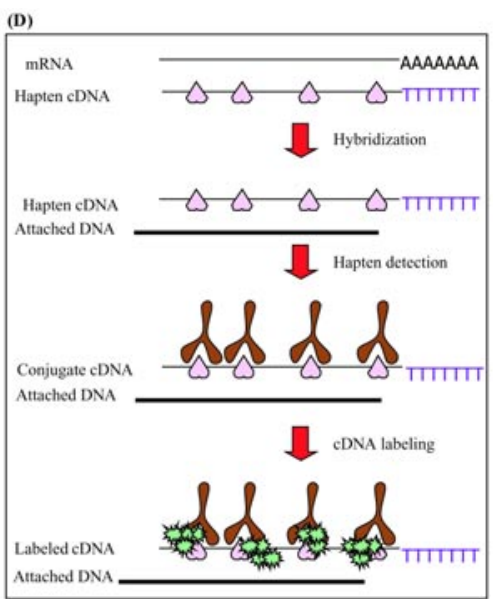

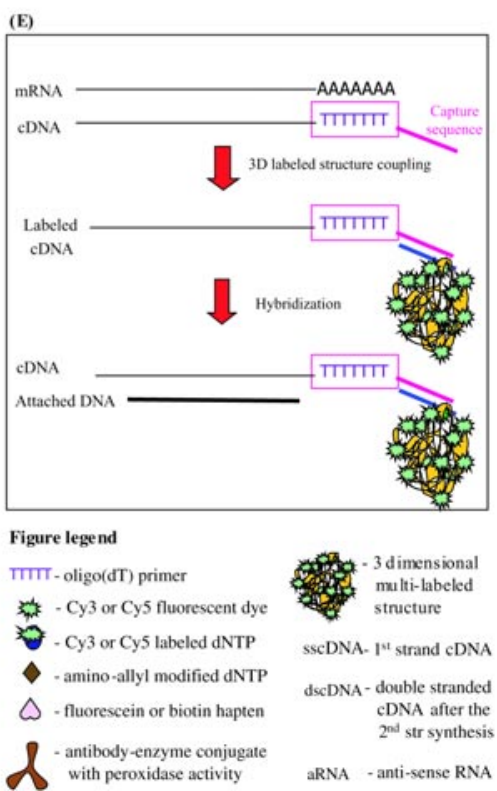

Figure 1. Scheme of different principles of fluorescent probe synthesis. (A) Direct labeling method. (B) T7 RNA polymerase amplification method. (C) Aminoallyl labeling method. (D) Hapten-antibody enzymatic labeling method. (E) Labeling method using 3-D multi-labeled structures.

antibody-enzyme conjugates recognize the fluorescein or biotin haptens. In a subsequent step, these conjugates catalyze the deposition of $\mathrm{Cy} 3$ and $\mathrm{Cy} 5$ dyes to the cDNA/attached DNA hybrids. The recommended starting material is $0.5-2 \mu \mathrm{g}$. In the labeling method using the 3-D multi-labeled structures (Figure 1E), a first-strand cDNA reaction is performed using a hybrid primer between oligo(dT) and a short "capture sequence". After cDNA synthesis, a 3-D structure with approximately $300 \mathrm{Cy} 3$ or Cy5 dye molecules is hybridized to the cDNA via a complementary sequence to the capture part on the $3^{\prime}$-end of each cDNA molecule. Only $1 \mu \mathrm{g}$ total RNA as starting material is needed.

\section{MATERIALS AND METHODS}

\section{Cell Culture and RNA Extraction}

The maintenance of cultured HeLa cells and treatment with hemin and desferrioxamine were performed as described (5). To evaluate the different labeling techniques, we chose a comparison of iron-loaded and iron-deficient cells because the differences in expression levels of some of the selected genes were expected to be more pronounced than a comparison between iron perturbed cells and an untreated control.

\section{Northern Blot Analysis}

Total RNA $(10 \mu \mathrm{g})$ from hemin- and desferrioxamine-treated HeLa cells was separated on a $1 \%$ formaldehyde agarose gel and transferred onto a nylon membrane (Nytran $\mathrm{N}^{\circledR}$; Schleicher \& Schuell, Dassel, Germany). The membrane was subsequently hybridized to radioactively labeled probes that correspond to those genes that were identified as regulated on the iron chip. Church buffer (7) was used for the hybridizations. The signals obtained were quantified on a fluoroimager (Fujifilm FLA2000; Amersham Biosciences, Piscataway, NJ, USA).

\section{Preparation of the Iron Chip Microarray Platform}

Clone selection, amplification, and purification. The genes that are immo- 


\section{Research Report}

Table 1. Primer Sequences Used for PCR Amplification of Spike-In and Negative Controls

\begin{tabular}{|c|c|c|c|c|}
\hline Control & Gene Name & Source Vector & Forward Primer $\left(5^{\prime} \rightarrow 3^{\prime}\right)$ & Reverse Primer $\left(5^{\prime} \rightarrow 3^{\prime}\right)$ \\
\hline \multirow{2}{*}{ Negative } & $\begin{array}{l}\text { Arabidopsis } \\
\text { unclassified protein }\end{array}$ & AL022198 & ACGATGAAGCATTGAGACTG & СССТССТTАСТTСТTСТGСAG \\
\hline & $\begin{array}{l}\text { Kanamycin } \\
\text { resistance fragment }\end{array}$ & $\begin{array}{l}\text { common plasmid } \\
\text { vectors }\end{array}$ & C12-ATGATTGAACAAGATGGATTGC & CGAATGGGCAGGTAGCCGGATC \\
\hline \multirow[t]{3}{*}{ Spike-In } & $\begin{array}{l}\text { Cytochrome } \\
\text { P450 exon } 3\end{array}$ & AL021687 & CACACCTTCAACGTTGCGTTGC & CGGCTTCTAGAACGTTGGGA \\
\hline & $\begin{array}{l}\text { Chloroplast omega- } 6 \text { fatty } \\
\text { acid desaturase, exon } 4\end{array}$ & AL022198 & GTTTGAGATTGATGATCTGA & ATTGCAGTTCCTGTCCATGC \\
\hline & $6 i 18 \_52 k$ & AL022198 & GGGGTAGACCTTTGATCG & TGTGACGACGATTGTATG \\
\hline
\end{tabular}

bilized on the iron chip were selected from $(i)$ the literature, (ii) microarray experiments performed on filters that contain approximately 20000 human non-redundant expressed sequence tags (ESTs) comparing hemin- and desferrioxamine-treated $\mathrm{CaCo} 2$ cells; and (iii) gene lists from published microarray studies that address metabolic pathways of interest. For the list of genes, see http://www.embl-heidelberg. de/ExternalInfo/hentze/suppinfo.html. Human EST clones corresponding to the selected genes were obtained from the German Resource Center [(RZPD) Berlin, Germany] and sequence verified from both ends. The ESTs were selected to contain the $3^{\prime}$-end of a cDNA (i.e., the polyadenylation signal) and to extend for at least $300 \mathrm{bp}$ towards the $5^{\prime}$ end. In addition, nine positive and negative controls were chosen. These are derived from bacterial and Arabidopsis genomes. All clones were PCR-amplified with 5'-amino-alkyl-modified vector primers using the Expand ${ }^{\mathrm{TM}}$ High Fidelity PCR System (Roche Applied Science, Rotkreuz, Switzerland). The PCR products were purified on a Biomek $^{\circledR} 2000$ robot (Beckman Coulter, Fullerton, CA, USA), using the NucleoSpin ${ }^{\circledR}$ Multi-96 Extract PCR purification system (Macherey-Nagel, Düren, Germany). After purification, $5 \mu \mathrm{L}$ of the purified reaction were subjected to quality control on $1 \%$ agarose gels. The size, quality (single vs. multiple bands), and concentration of the PCR frag- ments were determined. SSC buffer ( 3 $\mathrm{M} \mathrm{NaCl}, 0.3 \mathrm{M}$ sodium citrate, $\mathrm{pH}$ 7.0) $(20 \times)$ was added, resulting in a final concentration of $2 \times$ SSC. Nine microliters of the purified PCR products dissolved in $2 \times$ SSC were transferred to a 384-well plate for spotting.

Spotting and attachment. Silanized glass slides were used as a platform for spotting on the OmniGrid ${ }^{\mathrm{TM}}$ (GeneMachines ${ }^{\circledR}$, San Carlos, CA, USA) spotting robot. Split pins from TeleChem International (Sunnyvale, CA, USA) (spot size $120 \mu \mathrm{m}$ ) were used. All clones were spotted in triplicate next to each other. External controls and selected housekeeping genes (actin, gapdh, $\mathrm{H}$ $f e r$, and $L$-fer) were spotted in dilutions of $1: 1,1: 2$, and 1:4. For data quantitation (see Table 2), only the signals derived from the undiluted PCR products were used. Cy3-labeled oligonucleotides were spotted to control the performance of the spotting needles and DNA attachment quality in four of the eight resulting subgrids. Spotting was performed at $24^{\circ} \mathrm{C}$ with $60 \%$ relative humidity. After spotting, the slides were immediately placed in a $50^{\circ} \mathrm{C}$ humid oven for $3 \mathrm{~h}$ and additionally baked at $100^{\circ} \mathrm{C}$ for $10 \mathrm{~min}$ to stabilize DNA binding to the silanized glass surface.

\section{Prehybridization and Denaturation of Microarrays}

Slides that were used in hybridizations with fluorescent probes synthe- sized by all protocols were subjected to a prehybridization step. The microarrays were immersed at $42^{\circ} \mathrm{C}$ in $6 \times \mathrm{SSC}$, $0.5 \% \mathrm{SDS}, 1 \% \mathrm{BSA}$ for $40 \mathrm{~min}$ and subsequently washed briefly with double-distilled water at room temperature. Before hybridization, the spotted PCR products were always denatured by immersing the slides at $95^{\circ} \mathrm{C}$ in doubledistilled water for $2 \mathrm{~min}$. The excess liquid was removed from the slides by brief centrifugation at $715 \times g$ in a microplate centrifuge (model Z320; Hermle, Wehingen, Germany).

\section{Scanning and Data Analysis}

All microarrays were scanned on a GenePix ${ }^{\circledR}$ 4000B Microarray Scanner (Axon Instruments, Union City, CA, USA). For each labeling protocol, individual laser power and photomultiplier settings were used, allowing all signals to remain in the linear range of the scanner. Separate scan images for $\mathrm{Cy} 3$ and $\mathrm{Cy} 5$ were produced and analyzed using the ChipSkipper microarray data evaluation software (http: //pc-ansorge11.embl-heidelberg.de/ chipskipper). Intensity values for each spot were calculated by subtraction of the local background surrounding the spot. All spots were used for the calculation of a linear regression line. The regression line's parameters (offset and slope) were used for normalization. The resulting data were analyzed in Microsoft ${ }^{\circledR}$ Excel $^{\circledR}$. 


\section{Fluorescent Probe Synthesis}

Direct labeling method. Total RNA $(20 \mu \mathrm{g})$ from desferrioxamine- or hemin-treated HeLa cells was reversetranscribed with Oligo(dT) (12-18) Primer (Invitrogen, Carlsbad, CA, USA) using SUPERSCRIPT ${ }^{\mathrm{TM}}$ II Rnase $\mathrm{H}^{-} \mathrm{Re}-$ verse Transcriptase (Invitrogen) in the presence of $0.1 \mathrm{mM}$ Cy5-dUTP or Cy3dUTP (Amersham Biosciences), respectively. The labeling reactions and probe purifications were performed as described at http://cmgm.stanford.edu/ pbrown/protocols/4_human_RNA.html. Before hybridization, the purified Cy3and Cy5-labeled cDNAs were mixed, and $5 \mu \mathrm{g}$ Poly $(\mathrm{dA})$ and $1 \mu \mathrm{g}$ Human Cot1 DNA (both from Invitrogen) were added and subsequently evaporated in a Vacuum Concentrator 5301 (Vacufuge $^{\mathrm{TM}}$; Eppendorf ${ }^{\circledR}$, Hamburg, Germany) at $60^{\circ} \mathrm{C}$. The resulting pellet was dissolved in $12 \mu \mathrm{L}$ hybridization buffer (50\% formamide, $6 \times$ SSC, $0.5 \%$ SDS, $5 \times$ Denhardt's solution) and denatured by incubating at $95^{\circ} \mathrm{C}$ for $2 \mathrm{~min}$. The probe was then transferred onto the array under a $24 \times 24 \mathrm{~mm}$ coverslip and incubated in a humid chamber (GeneMachines) containing $2 \times$ SSC drops for providing humidity. Hybridization was performed for $12-16 \mathrm{~h}$ in a $42^{\circ} \mathrm{C}$ water bath (GFL, Burgwedel, Germany).

After hybridization, the microarrays were washed in $0.1 \times \mathrm{SSC}, 0.1 \% \mathrm{SDS}$ for $10 \mathrm{~min}$ and twice with $0.1 \times \mathrm{SSC}$ for 5 min on an orbital shaker (Certomat $\mathrm{H}^{\circledR}$; B. Braun, Melsungen, Germany), followed by a brief immersion of the slides in double-distilled water. All washing steps were performed at room temperature. Finally, the washed slides were dried by centrifuging them briefly at $715 \times g$ in a model Z320 microplate centrifuge (Hermle).

\section{T7 RNA Polymerase Amplification Method}

Total RNA $(5 \mu \mathrm{g})$ was introduced into a linear mRNA amplification protocol, as described at http://cmgm. stanford.edu/pbrown/protocols/amp protocol_3.html. Three micrograms of the T7 RNA polymerase amplified antisense RNA was subjected to a direct labeling reaction and hybridization as described earlier.

\section{Aminoallyl Labeling Method}

Total RNA $(15 \mu \mathrm{g})$ was subjected to a labeling reaction according to the Atlas $^{\mathrm{TM}}$ Glass Fluorescent Labeling Kit manual (BD Biosciences Clontech, Palo Alto, CA, USA), with the exception that cDNA synthesis was carried out at $45^{\circ} \mathrm{C}$ for $30 \mathrm{~min}$. Hybridization and washing protocols are described in the direct labeling protocol. The use of GlassHyb $^{\mathrm{TM}}$ Hybridization Solution (BD Biosciences Clontech) and conditions did not result in specific signals in our hands (data not shown).

\section{Hapten-Antibody Enzymatic Labeling Method}

Total RNA (1-2 $\mu \mathrm{g})$ was used as input material for probe preparation. cDNA synthesis, hybridization, and washing were performed exactly as recommended in the manual of the MICROMAX $^{\mathrm{TM}} \mathrm{TSA}^{\mathrm{TM}}$ Labeling and Detection Kit (fluorescein kit variant) (Perkin Elmer Life Sciences, Boston, MA, USA). Synthesized cDNA was purified by isopropanol precipitation. Poly(dA) $(5 \mu \mathrm{g})$ and $1 \mu \mathrm{g}$ Human Cot 1 DNA were added to cDNA probes before hybridization.

\section{Fluorescent Probe Synthesis Using 3-D Multi-Labeled Structures}

Total RNA $(1 \mu \mathrm{g})$ was subjected to probe preparation, hybridization, and washing as described in the manual of the $3 \mathrm{DNA}^{\mathrm{TM}}$ Submicro $^{\mathrm{TM}}$ Expression Array Detection Kit (Genisphere, Hatfield, PA, USA). One microgram of $\mathrm{Hu}$ man Cot1 DNA was added before hybridization of the cDNA to the microarray. The hybridization was performed in non-formamide buffer (vial 6) under a $24 \times 24 \mathrm{~mm}$ coverslip in a humid chamber at $65^{\circ} \mathrm{C}$ overnight.

\section{“Spike-In" and Negative Controls}

Spotted PCR products serving as positive or spike-in controls correspond to genes for which T7 RNA polymerase-synthesized RNAs can be added to the total input RNA. Five sequences were selected from Arabidopsis and bacterial genomes and PCR-amplified for spotting (Table 1): cytochrome P450 


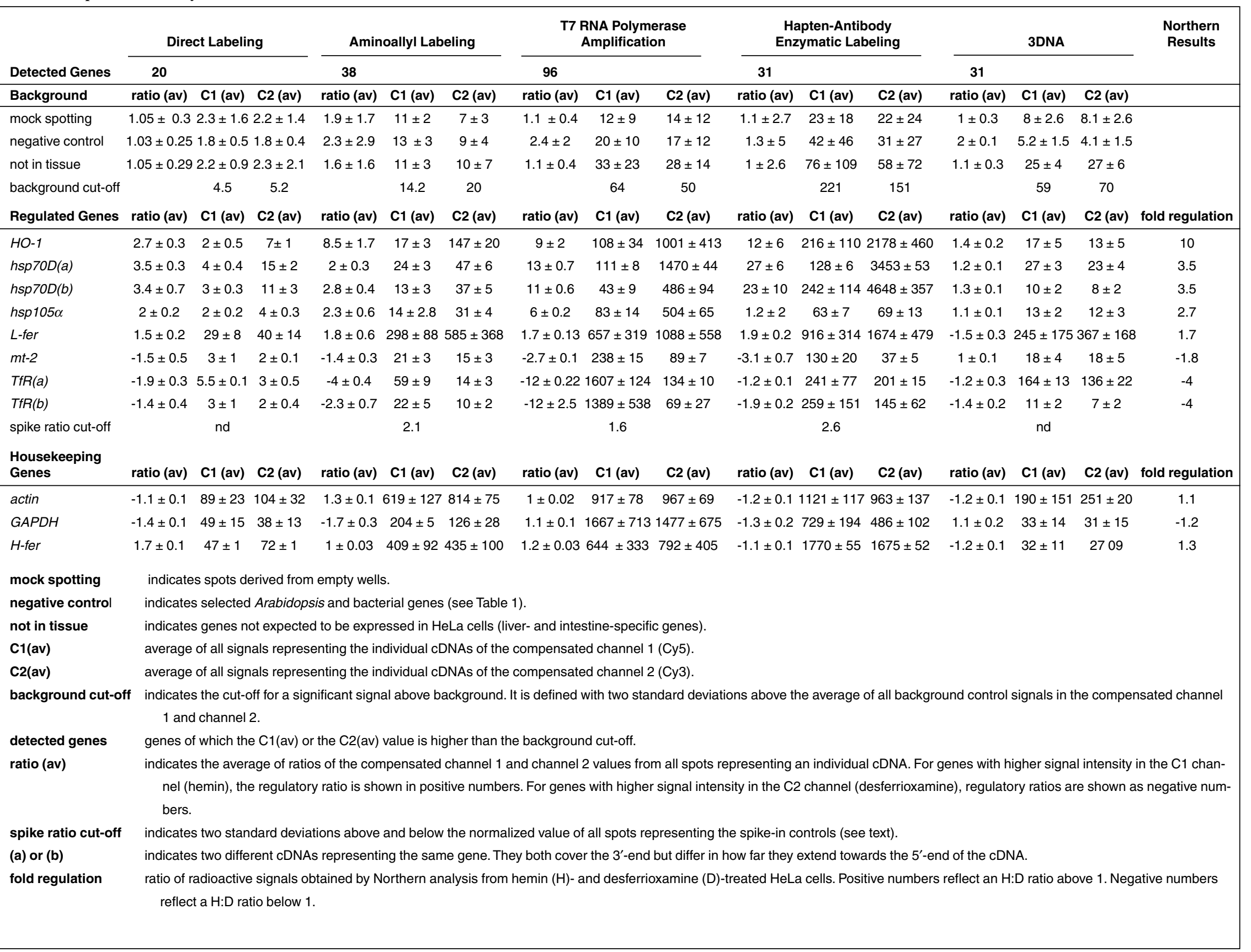


exon 3 (contained in GenBank ${ }^{\circledR}$ accession no. AL021687), chloroplast omega-6 fatty acid desaturase, exon 4 (contained in accession no. AL022198), $6 i 18 \_52 k$ (contained in accession no. AL022198), luciferase firefly (pt3LucpA) (6), CAT (pIRE-CAT-pA98) (6).

To produce dsDNA templates for the T7 RNA polymerase synthesis reactions of the spike-in controls, the following modifications were introduced into the PCR amplification primer set: the sequence of the T7 RNA polymerase promoter was added $5^{\prime}$ to the sequence of the forward primers, and an oligo dT(30) tail was added $3^{\prime}$ to the

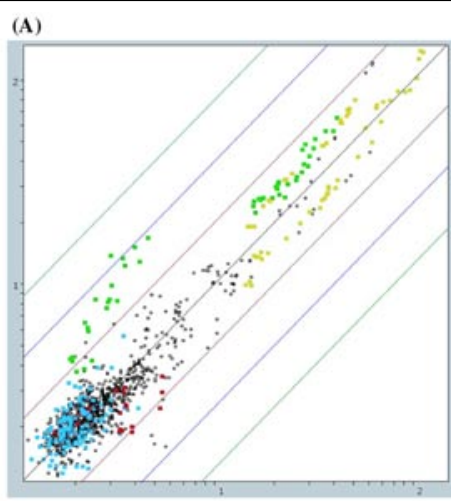

(C)

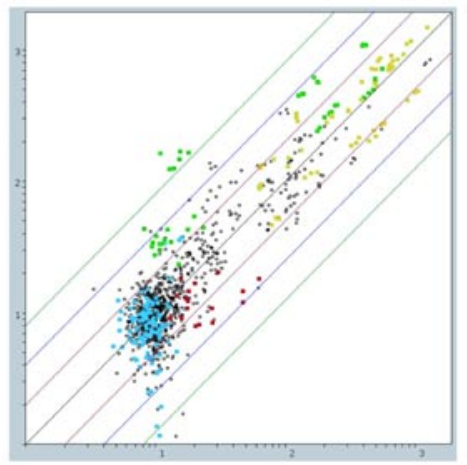

(E)

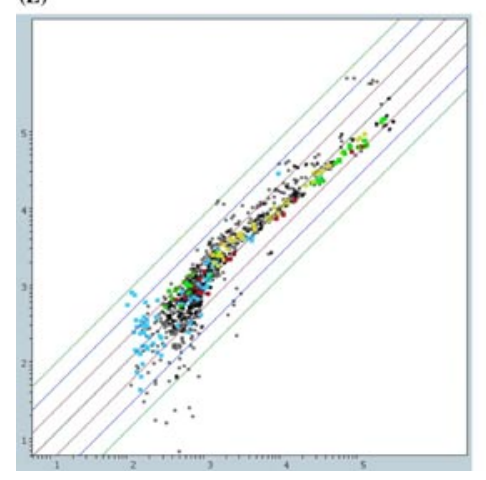

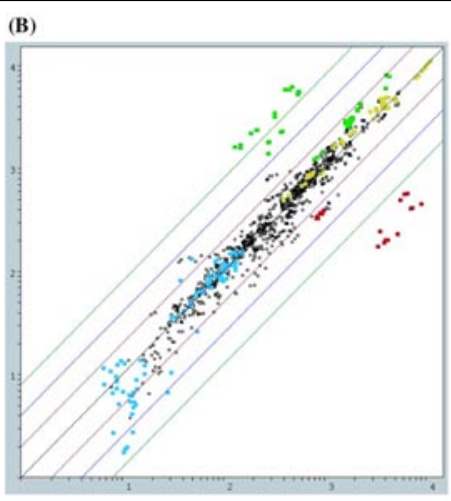

(D)

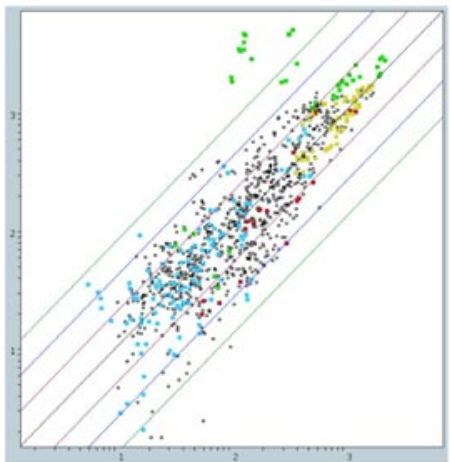

Figure legend

- regression line

- two-fold ratio line

four-fold ratio line

eight-fold ratio line

7. - negative signal spots (mock spots, DNA from different organisms, genes not expressed in HeLa cells)

- house-keeping genes

- genes overexpressed in hemin treated HeLa cells

- genes overexpressed in desferrioaxamine treated HeLa cells

- other genes

Figure 2. Scatter plot analysis. Fluorescent cDNA probes were synthesized using five different principles for fluorescent probe synthesis and hybridized to the iron chip, as described in the Materials and Methods section. Regulated genes, housekeeping genes, and negative controls are marked in colors as indicated. In the data shown, cDNAs derived from hemin-treated HeLa cells were labeled with Cy3 (yaxis) and from desferrioxamine-treated HeLa cells with Cy5 (x-axis). On this spotting batch of the iron chip, for the experiments performed with the hapten-antibody enzymatic labeling method, the cDNAs from hemin-treated HeLa cells were labeled with Cy5, and the cDNAs from desferrioxamine-treated cells were labeled with $\mathrm{Cy} 3$. For better illustration, the compensated $\mathrm{C} 1$ and compensated $\mathrm{C} 2$ channels are switched in the scatter plot. (A) Direct labeling method. (B) T7 RNA polymerase amplification method. (C) Aminoallyl labeling method. (D) Hapten-antibody enzymatic labeling method. (E) Labeling method using 3-D multi-labeled structures. 
Table 3. Summary of Pros and Cons of the Labeling Methods Tested

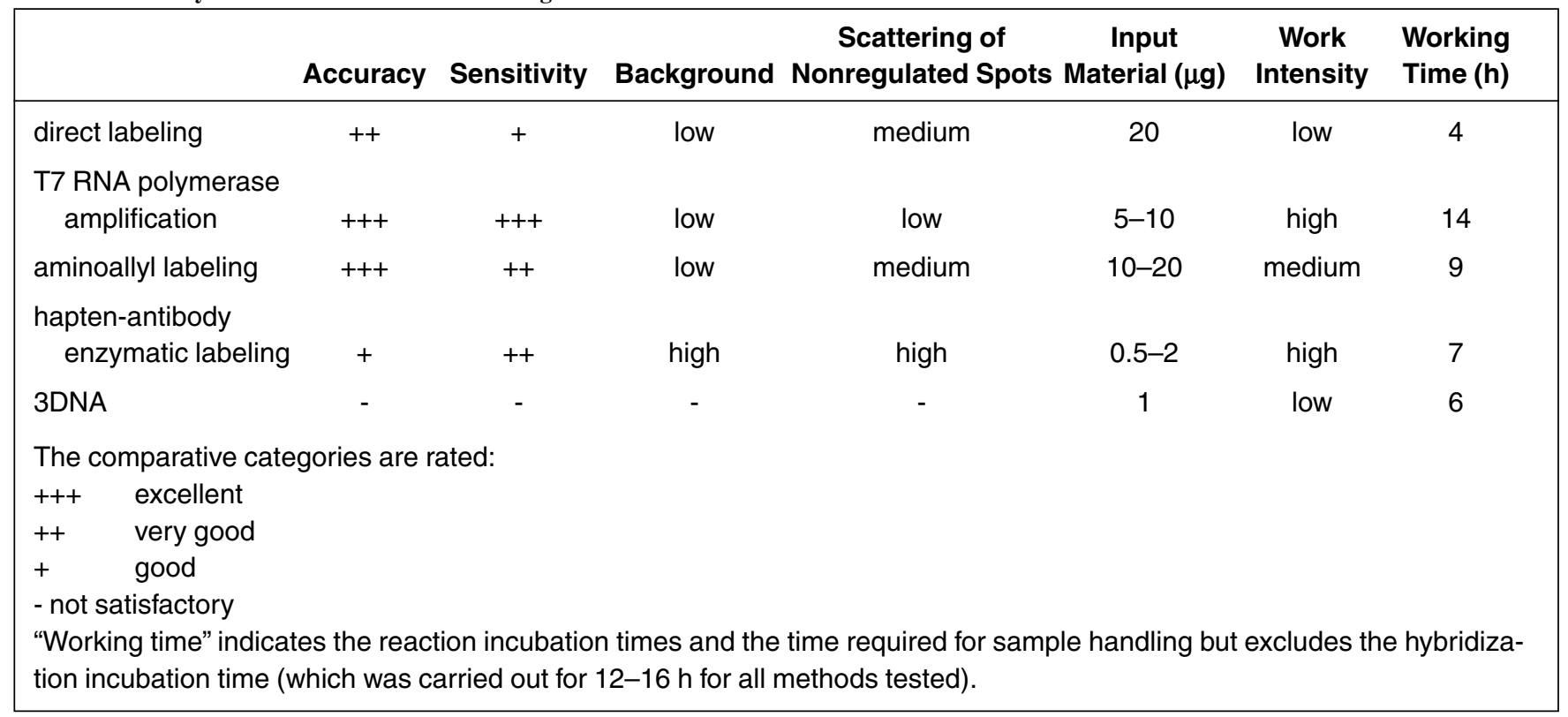

sequence of the reverse primers.

Spike-in RNAs are only useful in determining the cut-off for regulation if the signal intensities obtained from the spike-in control RNAs cover an intensity range that is similar to the signals obtained from cellular mRNAs. Thus, the amount of spike-in RNA has to be optimized for each of the labeling protocols. Fifty picograms of each spike-in RNA was added to both total RNA samples in the T7 RNA polymerase amplification method, and $100 \mathrm{pg}$ were used in the hapten-antibody enzymatic labeling protocol. One nanogram of each spike-in RNA was added to both input total RNA samples when the aminoallyl labeling protocol was used.

Negative control spots are used to determine the background noise of a microarray experiment. Four sequences were selected from Arabidopsis and bacterial genomes and PCR-amplified (Table 1): 8h10_70k (contained in accession no. AL133248), Arabidopsis unclassified protein (contained in accession no. A1022198), kanamycin resistance fragment commonly used in plasmid vectors, $p E G F P-N I$ (BD Biosciences Clontech) (accession no. U55762).

\section{RESULTS AND DISCUSSION}

To evaluate the different methods of fluorescent probe synthesis, we used the iron chip, a cDNA-based microarray system developed to investigate regulatory networks in iron metabolism. The 2.0 version of the iron chip used in this study is comprised of a selection of 120 genes that encode proteins that are directly involved in either iron metabolism or in one of the interlinked pathways, such as copper-metabolism, nitric oxide-metabolism, or the oxidative stress pathway. For the list of genes, see http://www.emblheidelberg.de/ExternalInfo/hentze/supp info.html. The genes of interest are represented by up to three cDNAs (up to three different ESTs) and are spotted in triplicate. Genes regulated in response to iron deficiency (desferrioxamine treatment) or iron exposure (hemin treatment) can be monitored in HeLa cells and be used to assess the dynamic range and signal-to-noise ratio of a microarray experiment.

To exclude experimental variability due to input sample variation, the same input RNA from iron-perturbed HeLa cells was used for all probe preparations. Furthermore, the cDNA arrays used for these comparative experiments are all derived from the same qualitycontrolled spotting batch. The results of the five different fluorescent labeling methods tested are shown as a scatter plot (Figure 2) and in tabular form (Table 2). Each experiment was per- formed at least twice; with the exception of the hapten-antibody enzymatic labeling method, the consistency of the obtained results was additionally ascertained by switching the fluorescent dyes between the hemin- and the desferrioxamine-treated cells. The scatter plots in Figure 2 represent cDNA from hemintreated cells (labeled with Cy3) on the $y$-axis and from desferrioxamine-treated cells (labeled with Cy5) on the $\mathrm{x}$ axis. Spots representing genes that are up-regulated in hemin-treated cells are depicted in green. Spots representing genes that are up-regulated in desferrioxamine-treated cells are depicted in red. Housekeeping genes are shown in yellow and negative controls in blue.

A comparison of the scatter plots reveals that the individual labeling methods differ with respect to the scattering of the nonregulated genes along the regression line (black line). This suggests that each of the methods yields different cut-off values for genes to be considered regulated.

The analysis of three of the experimental systems tested (aminoallyl labeling, T7 RNA polymerase amplification, and the hapten-antibody enzymatic labeling) was optimized by the addition of exogenous, mRNAs (spike-in controls) synthesized in vitro from heterologous organisms (bacteria and Arabidopsis) to the total RNA before cDNA synthesis (see Materials and Methods). We did not 
optimize the appropriate amount of spike-in material for the two other methods tested (direct-labeling and the labeling method using 3-D multi-labeled structures), as they proved to be either too insensitive or inaccurate. The spikein RNAs show comparable characteristics with respect to their scattering around their regression line as observed for the nonregulated experimental mRNAs (data not shown). The spike-in controls can help to define the cut-off value for regulation. This cut-off value is defined in the following way. First, equal amounts of spike-in RNAs are added to the total input RNA from hemin- and desferrioxamine-treated cells before cDNA synthesis. This means that the theoretical ratio for each individual spike-in RNA equals one. The signals of all spike-in controls in the $\mathrm{Cy} 3$ and the Cy5 channel are then quantified, and the sum of the signal intensities is normalized such that the experimental ratio of the combined spike-in controls also equals one. Finally, the deviation of each individual spike-in control from the normalized value is calculated and used to determine the standard deviation. We define the cut-off ratio for regulation in each microarray experiment as two standard deviations above and below one. This value is reflected in the spike ratio cut-off value in Table 2.

A key aspect addressed in this study is a comparison of the sensitivity of the different protocols tested. To define the set of genes that are detectable in a given experiment requires the prior determination of background for each of the methods evaluated. To assess the noise, we used two different types of negative controls: (i) mock spots (derived from empty plate wells) and (ii) negative hybridization controls containing heterologous DNAs derived from Arabidopsis or bacterial genomes. These control genes were selected from biochemical pathways that are not represented in mammalian organisms. In addition to these negative controls, we paid particular attention to human genes that are not expected to be expressed in a HeLa cell line (e.g., liver or intestine-specific genes). We consider a gene detectable and expressed if the signal intensity exceeds two standard deviations of the average signal intensity of all negative controls.
Applying this calculation to each of the methods tested, the expression signal of only 20 genes was detectable with the direct labeling method, the expression of 38 genes was detected with the aminoallyl labeling method, and the expression of 53 genes was detected with the hapten-antibody enzymatic labeling method. While for both the direct labeling method and the aminoallyl labeling method comparatively large amounts of total RNA ( 20 and $15 \mu \mathrm{g}$ ) were used, the hapten-antibody enzymatic labeling method yielded a higher number of detectably expressed genes with only $1 \mu \mathrm{g}$ total RNA. Thus, the hapten-antibody enzymatic labeling protocol can be considered significantly more sensitive than the other two. If a T7 RNA polymerase amplification step preceded the incorporation of fluorescently labeled nucleotides, then we could detect a specific signal from 96 of the cDNAs immobilized on the iron chip. This is close to the number of different genes represented on the iron chips that are expected to be expressed in HeLa cells.

We next assessed the quality of each of the methods in revealing the differential regulation of genes in response to iron availability. The observed ranges of regulation were compared to results obtained by Northern blot analysis for a selected set of genes. The fold regulation calculated from the Northern blot analysis is shown in Table 2 and in parentheses. Genes up-regulated in iron-deficient cells include the transferrin receptor (TfR) (4-fold) and metallothionine 2 (mt-2) (1.8-fold); genes up-regulated in iron-replete cells include heme-oxygenase 1 (HO- 1 ) (10-fold), L-ferritin ( $L$ fer) (1.7-fold), and the heat shock proteins (hsp) $70 D$ (3.5-fold) and $h s p 105 \alpha$ (2.7-fold). Housekeeping genes such as actin and GAPDH are not differentially expressed in iron-perturbed HeLa cells. $H$-ferritin ( $H$-fer) mRNA that is mainly regulated via a translational control mechanism in response to iron (3) also remains unchanged.

As can be seen in Figure 2 and Table 2 , the different methodologies to synthesize fluorescent probes reflect the expected regulatory responses to various degrees.

The direct labeling method identified the differential regulation of the HO-1, hsp $70 D$, and $h s p 105 \alpha$ genes, 
while the regulation of $T f R$ and $m t-2$ is variable between spotted triplets. A likely explanation for the latter is the low signal strength that hardly exceeds background. This method also could not resolve the weak regulation of the $L$-fer gene. Housekeeping genes like actin, GAPDH, and $H$-fer are not regulated, as expected (Figure 2A).

The T7 RNA polymerase amplification protocol that relies on linear mRNA amplification preceding the synthesis of labeled cDNA shows a highly pronounced regulation of the $H O-1$, $h s p 70 D$, hsp 105 $\alpha$, TfR-1, and $m t-2$ genes. It also reliably detects the weak regulation of the $L$-fer gene, as would be expected from the Northern analysis data. It should be noted that the signals for $T f R$ and $m t-2$ are clearly detected as being up-regulated in iron-deficient cells with this method, suggesting that the significantly increased signal-tonoise ratio allows this regulation to be observed. The nonregulated housekeeping genes (yellow spots in Figure 2) and the spike-in controls that were added to equal amounts to the total RNA from hemin- and desferrioxamine-treated cells before the amplification (data not shown) yield comparable signal intensities in the Cy3 and the Cy5 channel. They are located in close proximity to the regression line. The spike-in cut-off for regulation was calculated to be 1.6fold (Figure 2B).

With the aminoallyl labeling method, the regulation of the $\mathrm{HO}-\mathrm{l}$, $h s p 70 D$, and $h s p 105 \alpha$ genes was detectable. The regulation of $T f R$ was however only seen for those triplets that are above background. The regulation of $m t-2$ was not detected, and $L-f e r$ was only found to be regulated in two out of four triplets. The regulatory ratios obtained with this method most closely reflect the data obtained by Northern analysis. The housekeeping genes and the spike-in controls are more widely scattered around their regression lines in comparison to the T7 RNA polymerase amplification method. The spike-in cut-off for regulation was calculated to be 2.1 (Figure 2C).

Using the hapten-antibody enzymatic labeling method, we can detect the regulation of $H O-1$ and $h s p 70 D$ but not of the $h s p 105 \alpha$ gene. The signals for $T f R-1$ and $m t-2$ disappear in the widely scattered cloud of background spots or lie close to it. The advantage of the hapten-antibody enzymatic labeling protocol is the low input material requirement for probe synthesis; however, the wide scattering of the nonregulated spots and high background levels mask the regulation of genes that show lower signal-tonoise ratios and/or less pronounced regulation. We found it difficult to define a cut-off level for regulation. The spike-in cut-off for regulation was calculated to be 2.8 -fold (Figure 2D). 
In two separate experiments, we doubled the amount of input material (2 $\mu \mathrm{g})$ in comparison to the experiment shown here (data not shown). In this case, it was possible to detect the regulation of $T f R-1$ and $m t-2$. In addition, the nonregulated spots were less scattered around the regression line. However, most signals derived from highintensity spots contained a black center (usually the area of highest signal intensity). According to the manufacturer, this is due to a high density of assembling antibodies, which blocks the dyes from binding to the complex. The antibody directed against the fluorescein hapten apparently binds with higher affinity than the antibody directed against the biotin hapten because these high-intensity spots often are seen to be up-regulated in the Cy5 channel. These problems complicate the quantification of the data and significantly reduce the linear analytical range of an experiment, especially in the high-intensity range.

The labeling method with the 3-D multi-labeled structures was used according to the manufacturer's instructions, starting with $1 \mu \mathrm{g}$ total RNA input material. We obtained microarrays with intense signals and little background. However, analyzing the data, we were not able to reproduce the expected regulatory pattern as seen with the other methods and by Northern analysis. Genes that appear regulated have not been seen regulated with the other methods. Furthermore, while GAPDH, for example, is regarded as a highly expressed gene with all the other methods tested, it is barely detectable employing the protocol using 3-D multi-labeled structures. In our hands, this method failed to detect the expected gene expression pattern reliably. We cannot exclude the possibility that this method is incompatible with the chemical groups on the surface of our microarrays, although, according to the manufacturer's manual, the method should be compatible with "commercial or in-house microarrays" (Figure 2E).

\section{CONCLUSION}

We conclude that the choice of the cDNA labeling method can critically af- fect the outcome of a microarray experiment. Hence, we strongly recommend independent verification of gene regulatory patterns, at least initially, by, for example, Northern analysis, RNA protection assays, or quantitative RT-PCR. Most methods tested here were not capable to reveal the regulatory pattern fully. The reasons for this are the limited sensitivity and specificity, which result in the loss of the detection of expressed and/or regulated genes. Another reason is signal variability, which is reflected by the scattering of the nonregulated spots around the regression line. A high level of scattering makes it difficult to reveal low-level regulation.

For reasons that are not clear to us, the protocol using 3-D multi-labeled structures did not allow the detection of the gene expression pattern, despite the presence of high signals above background on the array. Alternative microarray platforms may possibly be more compatible with this method. The hapten-antibody enzymatic labeling method is sensitive but creates high levels of background. However, it may be useful in experimental systems with limited amounts of starting material and in which, for example, the T7 RNA polymerase amplification protocol cannot be applied because of a lack of poly(A) tails (e.g., bacteria). The direct labeling and the aminoallyl labeling methods are reliable technologies but require large amounts of starting material. These methods are less expensive than the T7 RNA polymerase amplification protocol and may be suitable alternatives when the quantity of starting material is not limiting (e.g., in cell culture experiments). For the reasons discussed earlier, the most sensitive and reliable method in our hands is based on linear T7 RNA polymerase-mediated mRNA amplification and subsequent cDNA synthesis. Wang et al. (9) also reported the high quality of this method. For a summary of the pros and cons of each of the fluorescent labeling methods, see also Table 3.

This comparison of different principles of fluorescent probe synthesis might help researchers to find their preferred cDNA labeling technique in the quickly growing field of microarray analysis. It also highlights potential problems that will arise for the compar- ison and interpretation of microarray data in public array databases that are currently being set up (2).

\section{ACKNOWLEDGMENTS}

Funds from the Gottfried Wilhelm Leibniz Award to M.W.H. were used to establish the iron chip, which is based on microarray technology. We thank the Resource Center and Primary Database (RZPD) for the supply of IMAGE clones.

\section{REFERENCES}

1.BD Biosciences Clontech. Atlas ${ }^{\mathrm{TM}}$ Glass Fluorescent Labeling Kit User Manual (PT34521). BD Biosciences Clontech, Palo Alto, CA.

2.Brazma, A., P. Hingamp, J. Quackenbush, G. Sherlock, P. Spellman, C. Stoeckert, J. Aach, W. Ansorge, et al. 2001. Minimum information about a microarray experiment (MIAME)-toward standards for microarray data Nat. Genet. 29:365-371.

3.Hentze, M.W., S.W. Caughman, T.A. Rouault, J.G. Barriocanal, A. Dancis, J.B. Harford, and R.D. Klausner. 1987. Identification of the iron-responsive element for the translational regulation of human ferritin mRNA. Science 238:1570-1573.

4.Kallioniemi, O.P. 2001. Biochip technologies in cancer research. Ann. Med. 33:142-147.

5.Muckenthaler, M., N. Gunkel, R. Stripecke, and M.W. Hentze. 1997. Regulated poly(A) tail shortening in somatic cells mediated by cap-proximal translational repressor proteins and ribosome association. RNA 3:983-995.

6.Preiss, T., and M.W. Hentze. 1998. Dual function of the messenger RNA cap structure in poly(A)-tail-promoted translation in yeast. Nature 392:516-520.

7.Sambrook, J., E.F. Fritsch, and T. Maniatis. 1989. Molecular Cloning: A Laboratory Manual. CSH Laboratory Press, Cold Spring Harbor, NY.

8.Schulze, A. and J. Downward. 2001. Navigating gene expression using microarrays-a technology review. Nat. Cell Biol. 3:190-195.

9.Wang, E., L.D. Miller, G.A. Ohnmacht, E.T. Liu, and F.M. Marincola. 2000. High-fidelity mRNA amplification for gene profiling. Nat. Biotechnol. 18:457-459.

Received 19 March 2002; accepted 15 May 2002.

\author{
Address correspondence to: \\ Dr. Martina Muckenthaler \\ European Molecular Biology Laboratory \\ Meyerhofstrasse 1 \\ D-69117 Heidelberg, Germany \\ e-mail:muckenthaler@embl-heidelberg.de
}

